\title{
MAXIMA: an experiment to measure temperature anisotropy in the cosmic microwave background
}

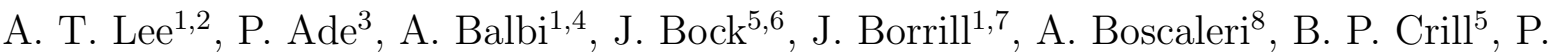 \\ De Bernardis ${ }^{9}$, H. Del Castillo ${ }^{6}$, P. Ferreira ${ }^{10}$, K. Ganga ${ }^{11}$, S. Hanany ${ }^{12}$, V. Hristov ${ }^{5}$, A. H.

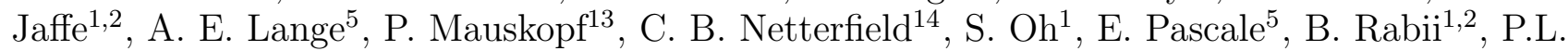

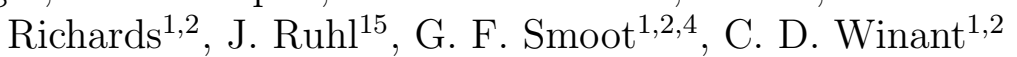 \\ ${ }^{1}$ Center for Particle Astrophysics, 301 LeConte Hall, University of California, Berkeley, CA 94720 \\ ${ }^{2}$ Department of Physics, University of California, Berkeley, CA 94720 \\ ${ }^{3}$ Queen Mary and Westfield College, London, UK \\ ${ }^{4}$ Lawrence Berkeley National Laboratory, Berkeley, CA 94720 \\ ${ }^{5}$ Department of Physics, California Institute of Technology, Pasadena, CA 91125 \\ 6 Jet Propulsion Laboratory, Pasadena, CA 91125 \\ 7 National Energy Research Scientific Computing Center, LBNL, Berkeley, CA 94720 \\ 8 IROE-CNR, Firenze, Italy \\ 9 Dipartimento di Fisica, Universita La Sapienza, Roma, Italy \\ 10 CERN, Geneve 23, Switzerland and CENTRA, Instituto Superior Tecnico, Lisboa 1096 Codex, Portugal \\ 11 IPAC, Pasadena, CA 91125 \\ 12 School of Physics and Astronomy, University of Minnesota, Minneapolis, MN 55455 \\ 13 University of Massachusetts, Amherst, MA, 01003 \\ ${ }^{14}$ Department of Physics, University of Toronto, Toronto, Canada \\ 15 Department of Physics, University of California, Santa Barbara, CA, 93106
}

\begin{abstract}
We describe the MAXIMA experiment, a balloon-borne measurement designed to map temperature anisotropy in the Cosmic Microwave Background (CMB) from $\ell=80$ to $\ell=800$. The experiment consists of a $1.3 \mathrm{~m}$ diameter off-axis Gregorian telescope and a receiver with a 16 element array of bolometers cooled to $100 \mathrm{mK}$. The frequency bands are centered at 150, 240, and $410 \mathrm{GHz}$. The 10' FWHM beam sizes are well matched to the scale of acoustic peaks expected in the angular power spectrum of the CMB. The first flight of the experiment in its full configuration was launched in August 1998. A $122 \mathrm{deg}^{2}$ map of the sky was made near the Draco constellation during the 7 hour flight in a region of extremely low galactic dust contamination. This map covers $0.3 \%$ of the sky and has 3200 independent beamsize pixels. We describe the MAXIMA instrument and its performance during the recent flight.
\end{abstract}

\section{INTRODUCTION}

Observations of the cosmic microwave background (CMB) temperature anisotropy are providing strong constraints on theories of cosmological structure formation. These measurements discriminate between competing cosmological models and, if the standard inflationary model is correct, will determine each many of the fundamental cosmological parameters to high precision.

In this paper we describe the MAXIMA experiment, a balloon-borne telescope designed to measure the angular power spectrum of CMB anisotropy over a wide range of angular scales. The experiment consists of a $1.3 \mathrm{~m}$ diameter off-axis Gregorian telescope and a receiver with a 16 element array of bolometers cooled to $100 \mathrm{mK}$. The high sensitivity of this receiver allows accurate measurements of the CMB power spectrum in a single overnight balloon flight. Each of the pixels in the array are sensitive to a single frequency band centered at 150, 240, or 410 GHz. The $150 \mathrm{GHz}$ band is the most sensitive to the CMB and is close in frequency to the predicted minimum in galactic foregrounds. The higher frequency channels monitor emission from the atmosphere and galactic foregrounds such as dust. The combination of a small beam size $\left(10^{\prime}\right.$ FWHM) and long scans $\left(4^{\circ} \mathrm{p}-\mathrm{p}\right)$ result in sensitivity from angular scales of $4^{\circ}$ to $10^{\prime}(80<\ell<800$, where $\ell$ is the spherical harmonic multipole number.) This range is well matched to the expected acoustic peaks in the CMB angular power spectrum. 
The scan strategy for MAXIMA has been carefully designed to be efficient and to minimize and measure systematic effects. The observation is done in a total power rather than chopped mode. The sky is scanned with three modulations: a primary mirror scan, a gondola scan, and the same patch of sky is scanned twice during the flight. These modulations allow interference on any one time scale to be isolated. The two scans of the sky are performed at different angles due to sky rotation to "cross link" the scans and reduce striping due to $1 / \mathrm{f}$ noise in the detector. This cross-linking results in a more sensitive measurement of the angular power spectrum.

The first science flight MAXIMA-1 was launched in August 1998. All systems worked well and bolometer loading and sensitivity were consistent with those estimated with ground-based measurements. A $122 \mathrm{deg}^{2}$ map of the sky was made near the Draco constellation during the 7 hour flight in a region of extremely low galactic dust contamination. This map covers $0.3 \%$ of the sky and has 3200 independent beamsize pixels.

MAXIMA is part of the MAXIMA/BOOMERanG collaboration (see papers by P. De Bernardis and S. Masi in these proceedings). MAXIMA and BOOMERanG share a common base of technologies, including bolometric detectors, optical filters, and attitude control systems. MAXIMA is optimized for multiple single-day flights whereas BOOMERanG is designed for a long duration flight around the antarctic continent.

MAXIMA is a valuable testbed for the technologies planned for the High-Frequency Instrument (HFI) of the Planck Explorer. The $100 \mathrm{mK}$ "spiderweb" bolometers built at JPL are similar to those planned for the HFI. [5] MAXIMA has a similar beamsize to the HFI at $150 \mathrm{GHz}$ and also uses single-color feedhorns in the focal plane.

In the following sections, we will describe the optical system, receiver design, detector system, gondola, attitude control system, scan strategy, calibration strategy, and performance during the recent flight.

\section{OPTICAL SYSTEM}

The MAXIMA telescope is an underfilled $\mathrm{f} / 1$ off-axis Gregorian design with two cold reimaging mirrors as shown in Fig. 1. The primary mirror is a $1.3 \mathrm{~m}$ diameter off-axis section of a paraboloid. The secondary and tertiary mirrors $(21 \mathrm{~cm}$ and $18 \mathrm{~cm}$ in diameter respectively) are off-axis ellipsoids with aspheric components which compensate the part of the aberrations introduced by the primary mirror. This optical system provides a $1^{\circ} \mathrm{x} 1^{\circ}$ diffraction-limited field-of-view at $150 \mathrm{GHz}$. A baffle between the two reimaging mirrors and a Lyot stop provide excellent telescope sidelobe performance. The overall design has many elements in common with the DIRBE telescope for the same reasons. The reimaging optics and all baffles are maintained at LHe temperature to reduce the optical power loading on the bolometers, thereby improving sensitivity. The non-optical interior surfaces of the box are covered with a mm-wave absorbing material [1]. A $1 \%$ neutral-density filter can be inserted in the optical path at the intermediate focus for tests using $300 \mathrm{~K}$ loads. The neutral-density filter is inserted and removed using a bellows-sealed screw on the outside of the cryostat.

The focal plane contains 16 single-color feedhorns designed for 10' FWHM beamsize as shown in Figs. 2-4. The focal plane is organized into four equal elevation rows each containing two $150 \mathrm{GHz}$ feeds, a $240 \mathrm{GHz}$ feed, and a $410 \mathrm{GHz}$ feed. Two types of feedhorn are used in the focal plane. For the $150 \mathrm{GHz}$ channels conical horns are used, since these channels are at the diffraction limit. The conical horns are smooth walled for ease of fabrication. The sidelobe performance of the horn is not critical given the use of a Lyot stop. For the 240 and $410 \mathrm{GHz}$ channels, Winston horns are used since these channels detect multiple optical modes.

The frequency bands are defined by metal-mesh filters built by the QMWC group. These are positioned inside light pipes between the feedhorns and the bolometers as shown in Fig. 4. For the $150 \mathrm{GHz}$ channels a high-pass filter is formed by a small circular waveguide at the back of the feedhorn. Spectra measured before the flight are shown in Fig. 5. High-frequency out-of-band leaks are dangerous due to the rapidly rising spectrum of the atmosphere. Three low-pass filters (two metal mesh and one made from alkali-halide powder in polyethylene) are used to block resonant leaks in the band-defining filters and to reduce optical loading on the cryogens. We tested for leaks above the passband for each channel by comparing bolometer response with and without a thick-grill high-pass filter blocking a chopped source. The cutoff frequency of the thick-grill filter was chosen to be just above the upper edge of the passband. Leak performance was found to be greatly improved in channels with two metal mesh filters (see Fig. 4) by introducing an absorbing section of lightpipe made from G-10 in between the two filters. The inner diameter of this lightpipe was chosen to be the same as that of the adjacent lightpipe. This technique, which may suppress multiple reflections between the filters, did not adversely affect in-band efficiency.

The feedhorns are supported by an aluminum plate at LHe temperature. Another plate, at $100 \mathrm{mK}$, supports the filter and bolometer assemblies. The two plates are separated by a $0.5 \mathrm{~mm}$ gap. The $100 \mathrm{mK}$ side of the gap is covered with absorber to prevent stray light from entering [1].

The bolometers are housed in integrating cavities fed by conical horns. Several different backshort geometries were evaluated including a flat plate $1 / 4 \lambda$ from the bolometer, a spherical cavity, and a sloping flat backshort. System 


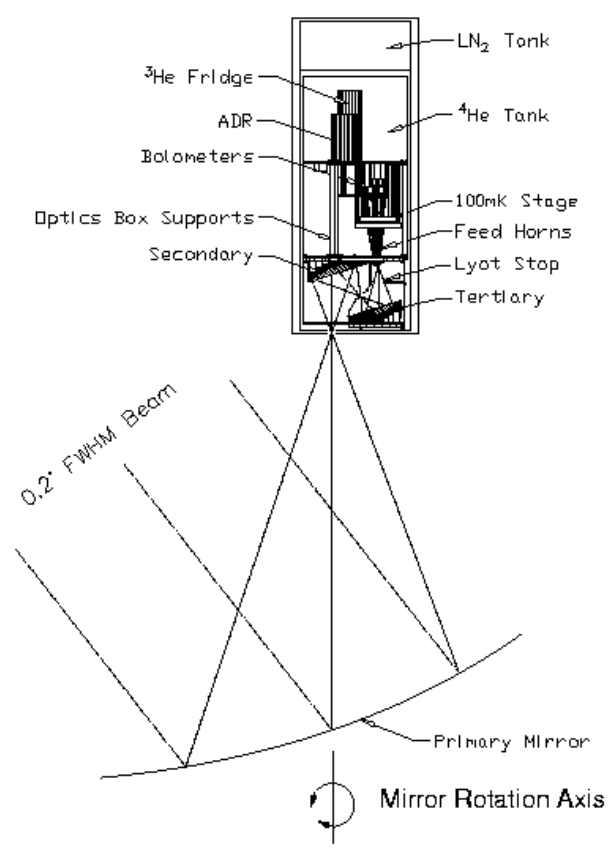

FIGURE 1. Cross-section of optical system. The primary mirror is a $1.3 \mathrm{~m}$ diameter off-axis paraboloid. The two reimaging mirrors are housed in a well baffled box that is maintained at LHe temperature. The optical system provides a $1^{\circ} \mathrm{x} 1^{\circ}$ diffraction-limited field-of-view at $150 \mathrm{GHz}$. A baffle at the intermediate focus and a Lyot stop provide excellent telescope sidelobe performance. The bolometers are cooled to $100 \mathrm{mK}$ by an Adiabatic Demagnetization Refrigerator. Both LN and LHe cryogen hold times are $\approx 40$ hours. The optical entrance to the receiver is vacuum sealed with a window made from 40 $\mu \mathrm{m}$ thick polypropylene.

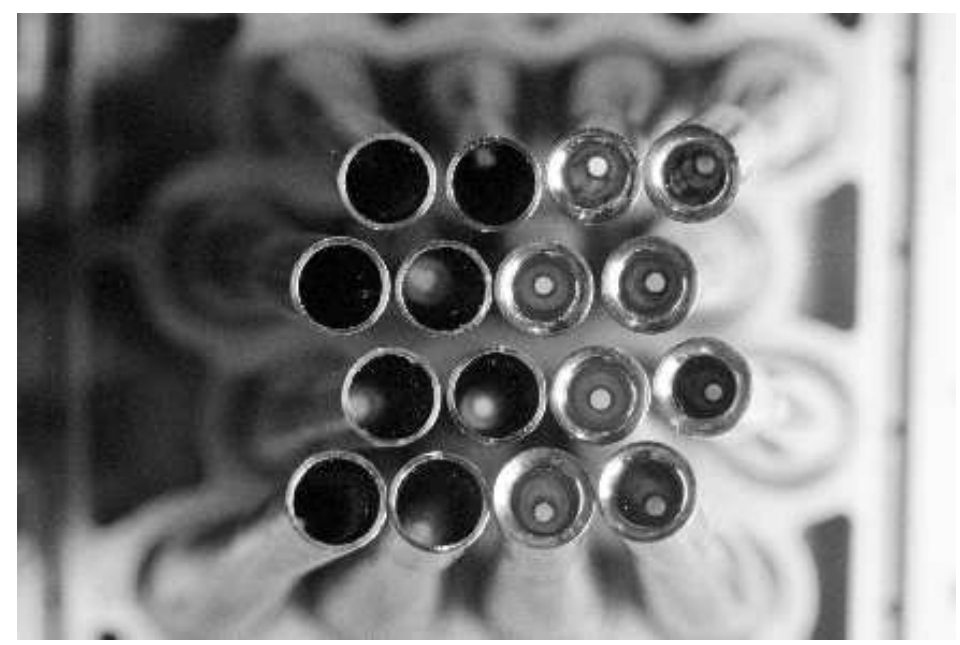

FIGURE 2. Photograph of MAXIMA focal plane. Smooth walled conical horns are used for the eight diffraction-limited $150 \mathrm{GHz}$ channels (two columns to left), and Winston horns are used for the 240 and $410 \mathrm{GHz}$ channels (two columns to right) which detect multiple optical modes. As the array is scanned in azimuth (horizontal in photo), each pixel is measured with four detectors in a row with the three frequency bands. The beamsize for all pixels is $10^{\prime}$. The outer diameter of the horns is $\approx 5.8 \mathrm{~mm}$.

optical efficiencies of most channels were 25-35\%, with no significant differences between the different backshort geometries.

The beams are scanned on the sky by rotating the primary mirror about an axis defined by the center of the primary mirror and the prime focus. We use a lightweight $(11 \mathrm{~kg})$ mirror constructed with an aluminum honeycomb core, graphite facesheets, and a sputtered aluminum optical surface. The mirror is rotated by a large dc servo motor. 


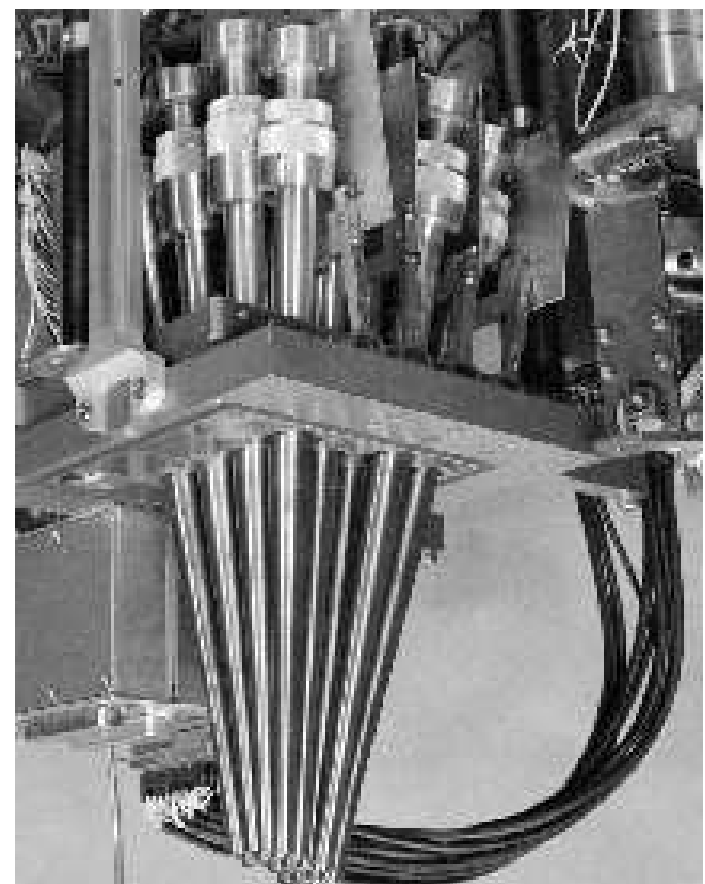

FIGURE 3. Photograph of the interior of the MAXIMA receiver. The focal plane is at the bottom with horns and light pipes extending to the LHe temperature mounting plate. The $100 \mathrm{mK}$ plate which carries the metal-mesh filter holders and bolometer integrating cavities is spaced $0.5 \mathrm{~mm}$ above the LHe plate. The ${ }^{3} \mathrm{He}$ refrigerator and heat switch used to conduct the ADR heat of magnetization to ${ }^{3} \mathrm{He}$ refrigerator are visible on the upper right. Each aluminum box (bottom left) contains FET front-end electronics for five channels.

The position is sensed by a Linear-variable-differential transformer (LVDT), and the position is controlled by an analog proportional-differential feedback system. The reference scan is a $0.45 \mathrm{~Hz} 4^{\circ}$ p-p sawtooth with smoothed transitions. Data from the flight is shown in Fig. 6.

Beam patterns were measured using Jupiter as a source during the MAXIMA-1 flight. Single azimuthal scans are shown in Fig. 7. for four channels. The beam sizes are consistent with the design goal of $10^{\prime}$ FWHM. Scans were performed at multiple elevations to measure the beam patterns in two dimensions.

The sidelobe performance was measured on the ground for the $150 \mathrm{GHz}$ band as shown in Fig. 8 . A $50 \mathrm{~mW}$ $150 \mathrm{GHz}$ Gunn oscillator source was placed on the roof of the NSBF highbay (height $\approx 37 \mathrm{~m}$ ) with the gondola in the parking lot in flight configuration. One telescope beam was pointed at the source and then the telescope was rotated first in elevation and then in azimuth to measure the sidelobe response. A variable attenuator was used on the source to keep the signal in the linear regime of the bolometers. In addition to the scans in elevation and azimuth the source was directed at all parts of the gondola from ground level.

\section{RECEIVER DESIGN}

The MAXIMA cryostat houses the secondary optics, the bolometers, and the sub-Kelvin coolers. [2] These are all mounted to a "cold plate" which forms one side of the LHe tank, and are surrounded by radiation shields at LHe and LN temperatures. The liquid nitrogen (liquid helium) tanks have a 13 (21) liter capacity, and the unpumped hold times are $\approx 40$ hours for both cryogens.

The bolometers are cooled to $100 \mathrm{mK}$ by an Adiabatic Demagnetization Refrigerator. A detailed description of this device can be found in Hagmann et al. [3] The salt pill is made from Ferric Ammonium Alum (FAA). The superconducting magnet uses a NbTi coil and generates $2.5 \mathrm{~T}$ for a peak current of 6.2 A. The magnet current is carried from the LN temperature stage to the LHe temperature stage by commercial leads made from High- $T_{c}$ superconductor, which give a heat leak of only $\approx 5 \mathrm{~mW}$. [4] The use of these leads gives a $15 \%$ decrease in total heat leak to the LHe compared to using normal metal leads. The cycle time of the ADR is 2 hours, and the hold time at $100 \mathrm{mK}$ is $\approx 48$ hours. The heat of magnetization is absorbed by a $300 \mathrm{mK}$ closed-cycle single-shot charcoal-pumped ${ }^{3} \mathrm{He}$ refrigerator, which also provides a heat intercept to the $100 \mathrm{mK}$ stage. The refrigerator contains $40 \mathrm{STP}$ liters 


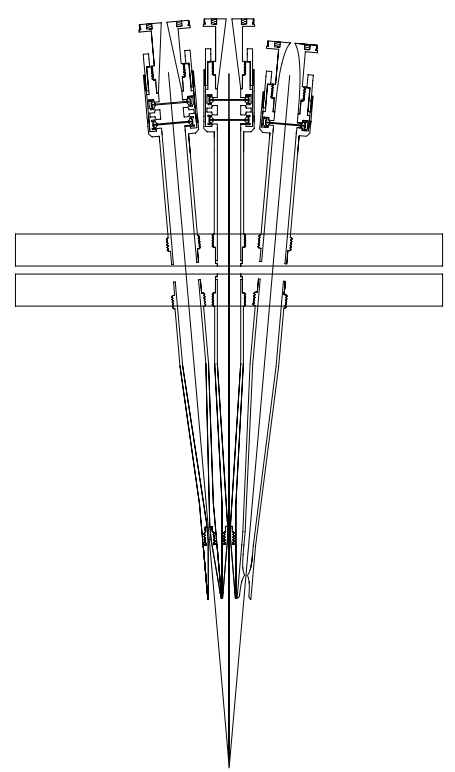

FIGURE 4. Cross-section view of the detector array. The single mode $150 \mathrm{GHz}$ channels (two horns on left) use smooth-wall conical feedhorns since they are diffraction limited to one optical mode. The 240 and $410 \mathrm{GHz}$ channels (only one horn shown on right) use Winston horns since they are sensitive to multiple optical modes. The frequency bands are defined by metal-mesh filters positioned within light pipes between the feedhorns and the bolometers. These filters are built by the QMWC group. For the $150 \mathrm{GHz}$ channels a high-pass filter is formed by a small circular waveguide at the back of the feedhorn. The feedhorns are mounted to a plate maintained at LHe temperature. The filters and bolometers are mounted to a $100 \mathrm{mK}$ plate that is separated by $0.5 \mathrm{~mm}$ from the LHe temperature plate. The bolometers and integrating cavities (not shown) are mounted behind the horns seen at the top of the drawing.

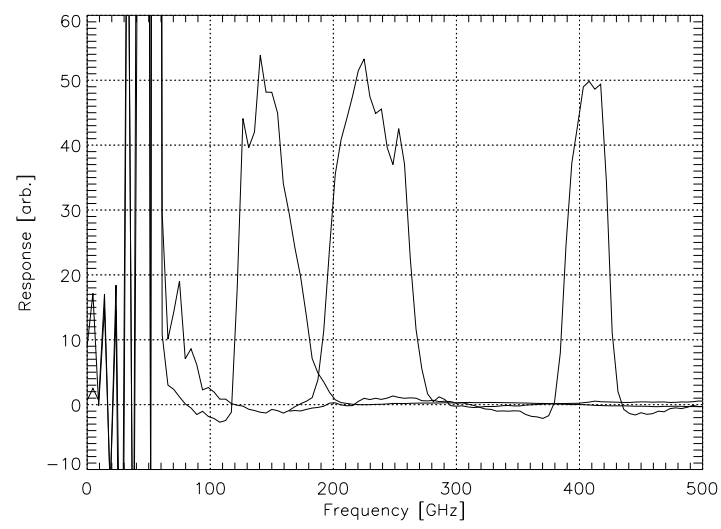

FIGURE 5. Spectral response of detector system. The data were obtained with a far-infrared Fourier spectrometer. The receiver was in flight configuration. The high noise below $100 \mathrm{GHz}$ results from the measurement method and does not reflect detector response.

of ${ }^{3} \mathrm{He}$ and uses an external tank to reduce the internal pressure when the system is at room temperature. The refrigerator takes 4 hours to cycle and has a hold time of 40 hours.

The heat leak to the $100 \mathrm{mK}$ stage has been minimized through the choice of materials and geometry of the supports and wires. The bolometer platform is mounted to the liquid helium cold plate with three tubes $(0.84 \mathrm{~cm}$ diameter, $17.8 \mathrm{~cm}$ long, and $0.5 \mathrm{~mm}$ wall thickness) made from Vespel SP-22 plastic which is strong but gives low heat leak at sub-Kelvin temperatures. The tubes are heat sunk to $300 \mathrm{mK}$ at $1 / 3$ of their length from the LHe temperature end. The $120 \mathrm{Pt}-\mathrm{W}$ bolometer wires are $5 \mathrm{~cm}$ long and have a 51 micron diameter. The signal wires are twisted with a ground wire and are attached to G-10 tubes (wrapped and varnished) to reduce microphonic response. The G-10 tubes are $10.2 \mathrm{~cm}$ long, $0.5 \mathrm{~cm}$ in diameter, have 130 micron wall thickness, and the heat flow 


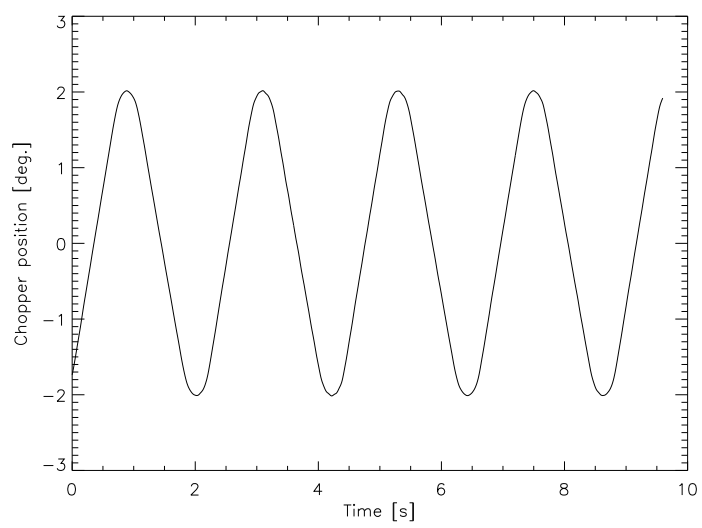

FIGURE 6. Measured azimuthal position of primary mirror during flight. The frequency is $0.45 \mathrm{~Hz}$ and the p-p scan is $4^{\circ}$ on the sky.

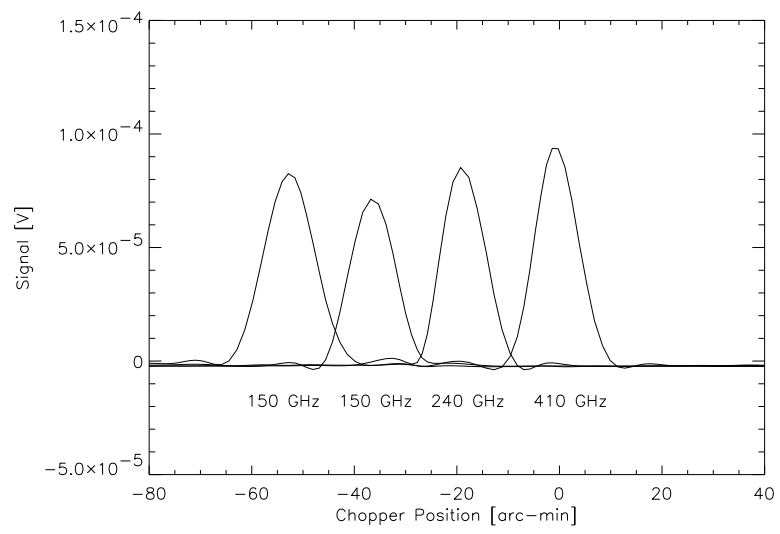

FIGURE 7. Beam patterns of one row of detectors using Jupiter as a source. Signals represent a single scan of the mirror in azimuth. Beam widths are 10-12' FWHM, without taking into account the finite size of Jupiter. There is some slight ringing in the baselines due to a combination of electronic and bolometer time constants. Detector noise is not visible on this plot due to the high signal-to-noise ratio.

is intercepted in a similar manner to the Vespel support legs.

We use a modular "wire insert" to carry all wires from the top of the cryostat at $300 \mathrm{~K}$ to the cold plate at LHe temperature. This insert, shown in Fig. 9, provides easy access to the wires. It is inserted through holes in the cryostat shell and the LN and the LHe tanks and is then mechanically attached at each temperature stage. It contains the $300 \mathrm{~K}$ connectors and essentially all of the cryostat wiring. It also includes cold RF filter modules made from striplines encased in lossy Eccosorb CR-124.

\section{DETECTOR SYSTEM}

We use spiderweb-absorber bolometers fabricated at JPL with Neutron-Transmutation Doped (NTD) Ge thermistors, which give high sensitivity with a fast response time, low cosmic-ray cross section, and low microphonic response. [5] The NTD Germanium material is produced at LBNL. Time constants of $\tau \approx 10 \mathrm{~ms}$ have been measured which are essential for our rapid primary mirror modulation. For our total power mode scan, the speed at which the beam can be modulated on the sky is limited to $\approx F W H M / 2 \tau$, where FWHM refers to the full-width half-maximum beamsize. [6]

The bolometer signals are buffered using differential JFET amplifiers. We use commercial devices from Infrared Laboratories that are internally thermally isolated with polyamide legs. [2] These devices dissipate $\approx 1 \mathrm{~mW}$ and have a white noise level of $\approx 9 n V / \sqrt{H} z$. 

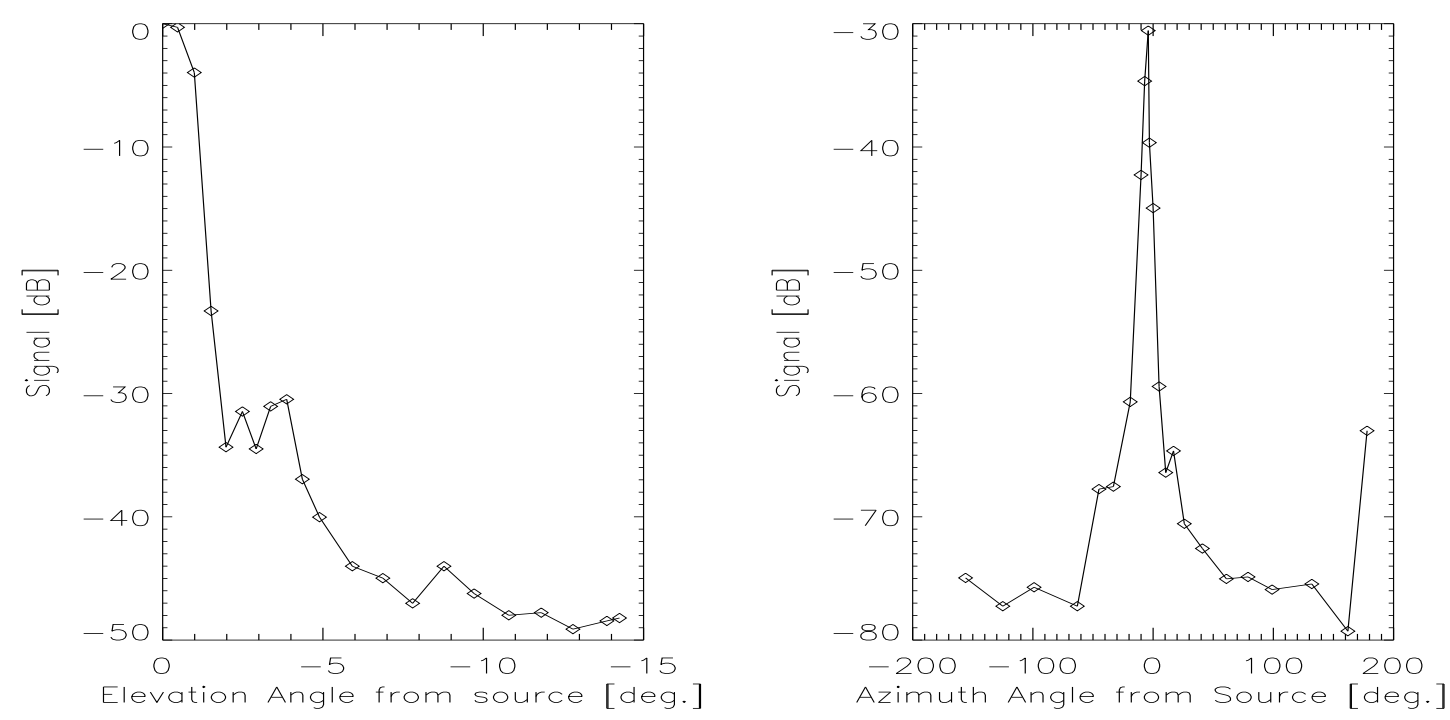

FIGURE 8. Sidelobe patterns for the MAXIMA telescope at $150 \mathrm{GHz}$. The near sidelobe pattern in elevation is at the left, and far sidelobe pattern in azimuth is at the right. Measurements were done with source on top of the NSBF highbay with a source elevation of $42^{\circ}$. Adjustable attenuators were used on the source to keep the detectors in the linear regime. The azimuth measurements were done with the elevation $\approx 2^{\circ}$ below the elevation of the source. This suppressed the main lobe during azimuth scan.

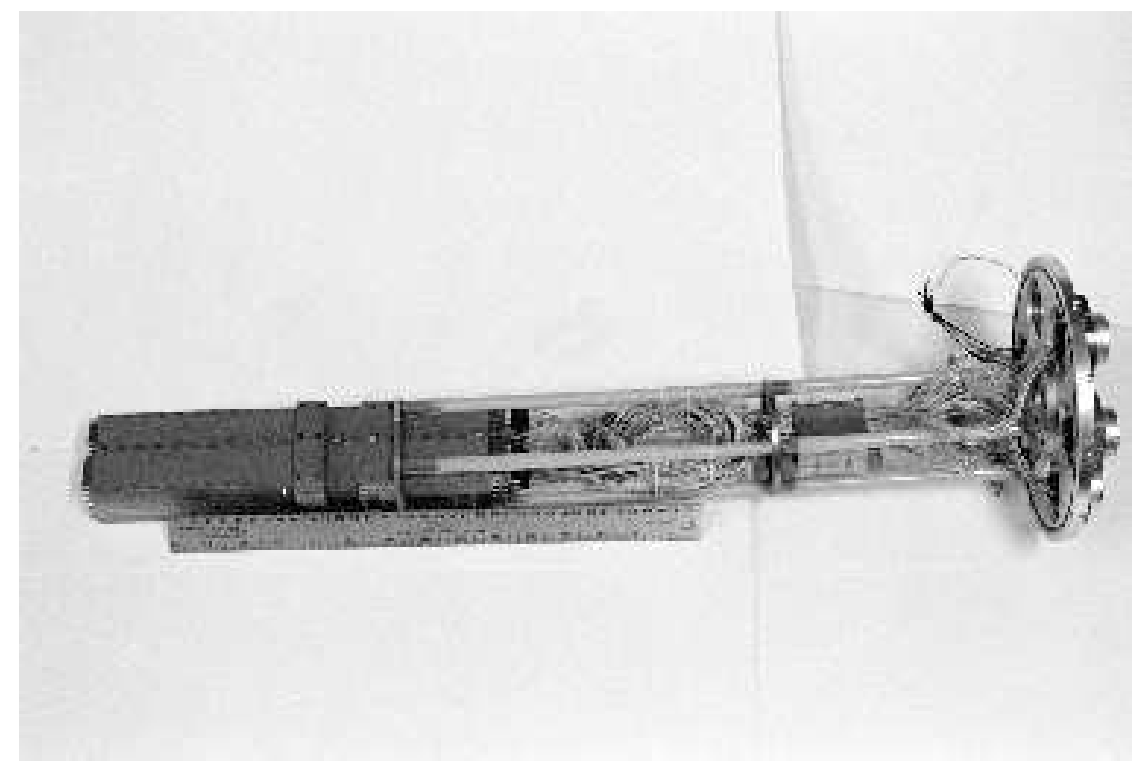

FIGURE 9. Photograph of wire insert. The modular wire insert is introduced through holes in the cryostat shell and LN and LHe tanks, and then mechanically attached to each temperature stage. The wire insert contains the $300 \mathrm{~K}$ connectors, essentially all of the cryostat wiring, and cold RF filter modules which consist of striplines encased in lossy Eccosorb CR-124. The three- and five-conductor stainless steel wires with braided stainless shields are organized into five wiring modules, one each for five bolometer channels, and one for housekeeping wires. Each of these modules can be easily replaced if found to be faulty.

The bolometers are ac-biased at $220 \mathrm{~Hz}$ using a sinusoidal bias and the signals are band-pass filtered and then lock-in detected. This ac-bias scheme circumvents $1 / \mathrm{f}$ noise in the front-end amplification and results in excellent low-frequency stability. [7,8] A noise spectral density from the flight is shown in Fig. 10 . The noise below $0.1 \mathrm{~Hz}$ is largely attributable to temperature fluctuations in the unregulated $100 \mathrm{mK}$ stage.

A $4.2 \mathrm{~K}$ temperature optical load was used in ground tests to check the optical loading without the neutral-density 


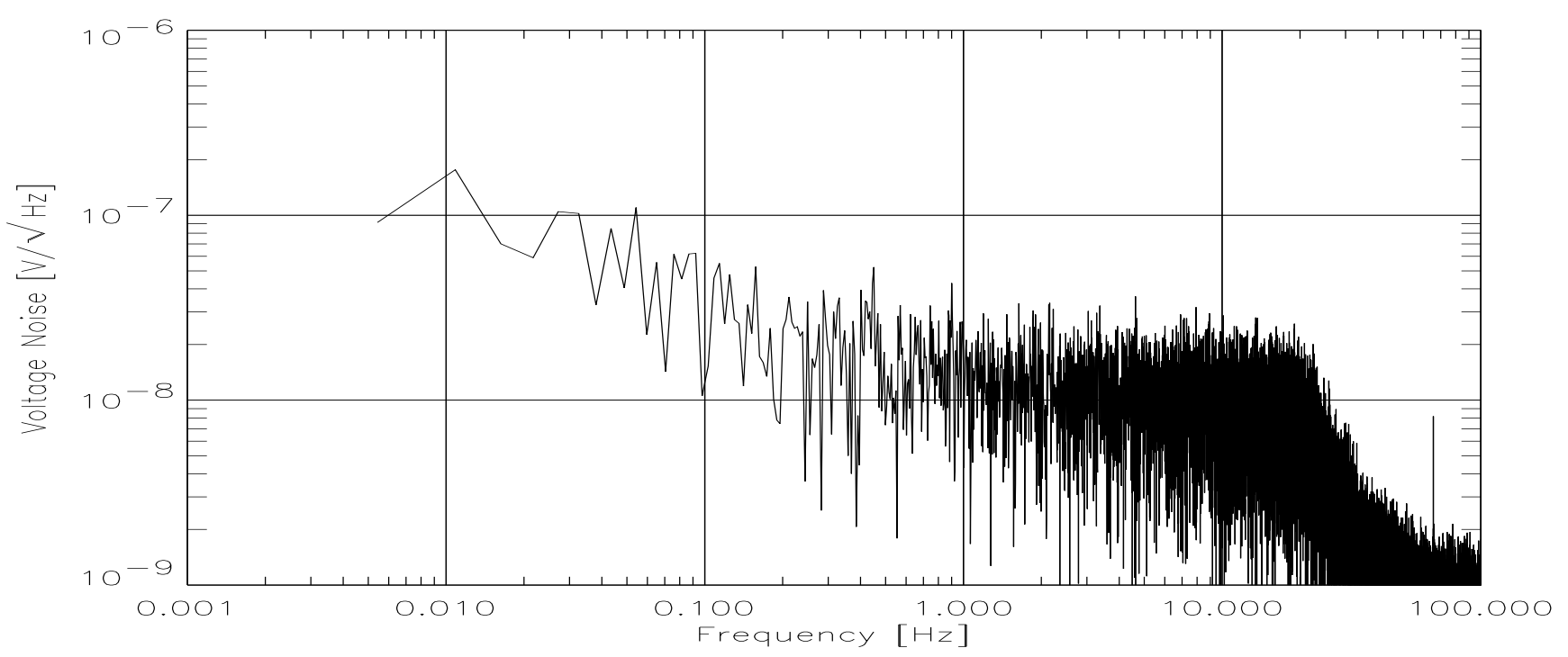

FIGURE 10. Noise referred to bolometer for $150 \mathrm{GHz}$ channel during CMB scan. The bolometers are ac-biased at $220 \mathrm{~Hz}$ using a sinusoidal bias and the signals are band-pass filtered and then lock-in detected. The effect of the $20 \mathrm{~Hz}$ low-pass filter in the readout electronics can be seen to the right. Low-frequency noise is largely attributable to temperature fluctuations in the unregulated $100 \mathrm{mK}$ stage.

filter. The optical loading on the bolometers in flight was consistent with that predicted from these measurements. During the MAXIMA-1 flight four of the eight $150 \mathrm{GHz}$ channels had a Noise Equivalent Temperature (NET-CMB) of 90-100 $\mu K \sqrt{s e c}$. Two others had NET $\approx 150 \mu K \sqrt{s e c}$, and the last two had significantly higher noise. The variability in sensitivity reflects a variation in noise more than in responsivity. Some excess noise came from the ac-bias readout system. The sensitivity of all $150 \mathrm{GHz}$ channels taken together in quadrature, which is the effective receiver sensitivity at $150 \mathrm{GHz}$, was $41 \mu K \sqrt{s e c}$.

We employ an optical "stimulator" located adjacent to the Lyot stop to provide a periodic calibration reference for the bolometers. This stimulator is similar to a bolometer in construction and uses a resistive heater to heat it to several tens of degrees Kelvin. It is turned on for 10 seconds every 25 minutes. We use three monitor channels in addition to the 16 optical bolometers. These include an optically blanked bolometer and a $4 \mathrm{MOhm}$ resistor, which are used to monitor electro-magnetic interference. We also use a well heat sunk NTD thermistor to monitor temperature fluctuations of the $100 \mathrm{mK}$ stage.

\section{GONDOLA AND ATTITUDE CONTROL SYSTEM}

The gondola, shown in Fig. 11, is designed to be strong enough for multiple flights. It incurred little damage in the MAXIMA-0 test flight in 1995 or in MAXIMA-1. The design is based on the ACME gondola used with the MAX experiment and is largely constructed with bolted aluminum. The gondola has a sturdy outer frame made from members with an "L" shaped cross-section, with tubular roll bars mounted at the corners. The receiver and primary mirror are mounted on an inner frame supported on trunion bearings to change the beam elevation. The elevation range is $20^{\circ}$ to $55^{\circ}$. The attitude control electronics, data acquisition, and command electronics are housed in boxes with aluminum honeycomb sides on the either side of the gondola. The bottom of the gondola is covered in aluminum honeycomb. The flight ready gondola weights 3600 lbs without ballast.

The attitude control system (ACS) controls the position of the telescope beams on the sky. The ACS consists of attitude sensors, a central feedback loop computer, and actuator motors. The ACS can be divided into one system that controls the azimuthal angle of the gondola and another that controls the elevation of the inner frame. For both systems, only some attitude sensors are used in the actuator control loop, while all are used for post-flight pointing reconstruction.

The azimuthal control portion of the ACS employs a pivot unit which contains two dc motors. One motor drives a reaction wheel and the other drives directly against the flight line. All scans are done with constant gondola azimuthal velocity. The feedback for the reaction wheel is proportional to azimuthal velocity as measured by an 


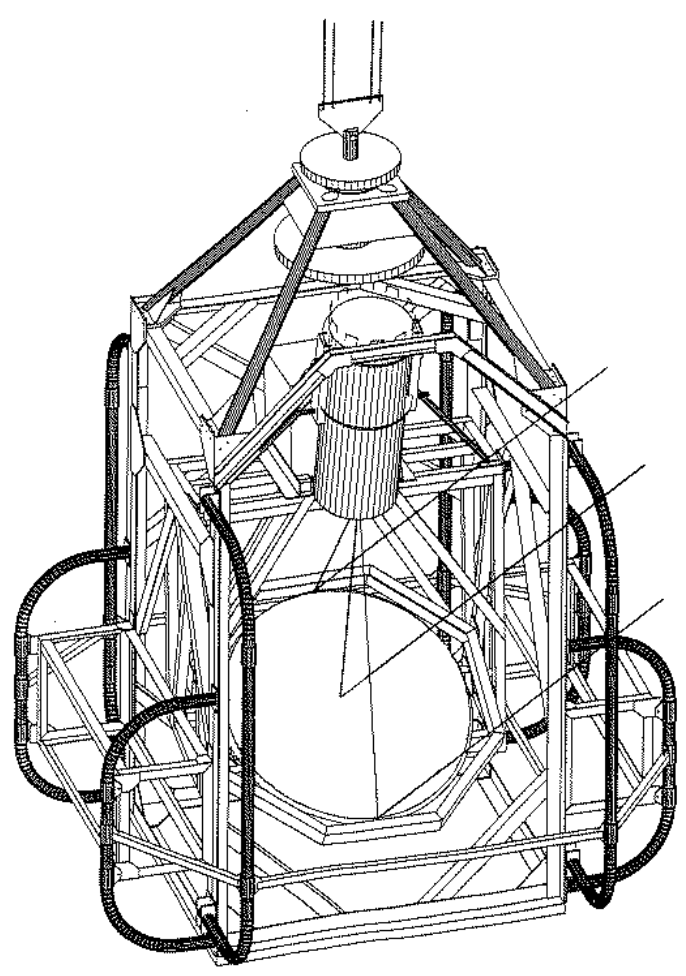

FIGURE 11. Drawing of the MAXIMA gondola. The primary mirror and the receiver are mounted to an inner frame supported on trunion bearings to change beam elevation from $20^{\circ}$ to $55^{\circ}$. The gondola is largely constructed with bolted aluminum members. The attitude control and data acquisition electronics are housed in the two aluminum boxes on the sides of the gondola.

azimuthal rate gyroscope. The feedback for the direct drive motor is proportional to the reaction wheel velocity as measured by a tachometer. This loop prevents the reaction wheel velocity from reaching saturation.

For the CMB anisotropy scan, the velocity is reversed periodically to produce a sawtooth scan. A two-axis magnetometer is used to determine the turnaround locations. During the MAXIMA-1 flight azimuthal velocity error was typically $\approx 0.3^{\prime} / \mathrm{s}$ with velocities during the anisotropy scans of $0.3-0.5^{\circ} / \mathrm{s}$. This performance is more than adequate since small errors in gondola velocity only affect the uniformity of sky coverage.

The telescope elevation is changed using a ballscrew and a small dc servo motor. The position is sensed using a 16-bit optical encoder. A digital feedback loop similar to the azimuth loop is used for control. The telescope elevation is not actively controlled during CMB scans. The telescope has a range in elevation of $20^{\circ}$ to $55^{\circ}$.

For post-flight pointing reconstruction of the CMB scans, the azimuth and elevation of the beam are detected with two CCD cameras. The boresight camera has a $7^{\circ}$ field of view in azimuth, and can lock on stars as faint as 6 th magnitude. The other camera is attached to the outer frame, has a $14^{\circ}$ field of view, and is offset in azimuth to lock on Polaris during the CMB scans. The two-axis magnetometer is used for pointing reconstruction during azimuthal rotations which were used to calibrate from the CMB dipole. Roll and pitch rate gyroscopes are also used in the pointing reconstruction.

Gondola pendulations give signals in the bolometers due to the change in optical path length through the atmosphere with beam elevation. The most troublesome mode observed during the 1995 MAXIMA-0 test flight was the one in which the gondola rotates about its center of mass. [9] For our gondola, the frequency of this mode is 0.6 $\mathrm{Hz}$, which is in our signal bandwidth. The lower frequency modes are not important during the CMB scans, since they occur at frequencies much lower than our $0.45 \mathrm{~Hz}$ mirror scan. Four approaches were used to reduce the 0.6 $\mathrm{Hz}$ pendulation. First, the reaction wheel was made as symmetric as possible by using well measured weights at the end of lightweight spokes. Second, the axis of the reaction wheel was aligned to the gravity vector to an accuracy of $\approx 1^{\prime}$ before flight. Third, the azimuth feedback loop uses a sensor (gyroscope) that has no sensitivity to pitch or roll. Finally, we use a passive pendulation damper built by Geneva Observatory, which consists of a damped harmonic oscillator whose resonance frequency is set to the $0.6 \mathrm{~Hz}$ pendulation frequency. [10] This device is constructed from a spherical weight rolling on a spherical cup in an oil filled vessel. We calculate that this damper should reduce 


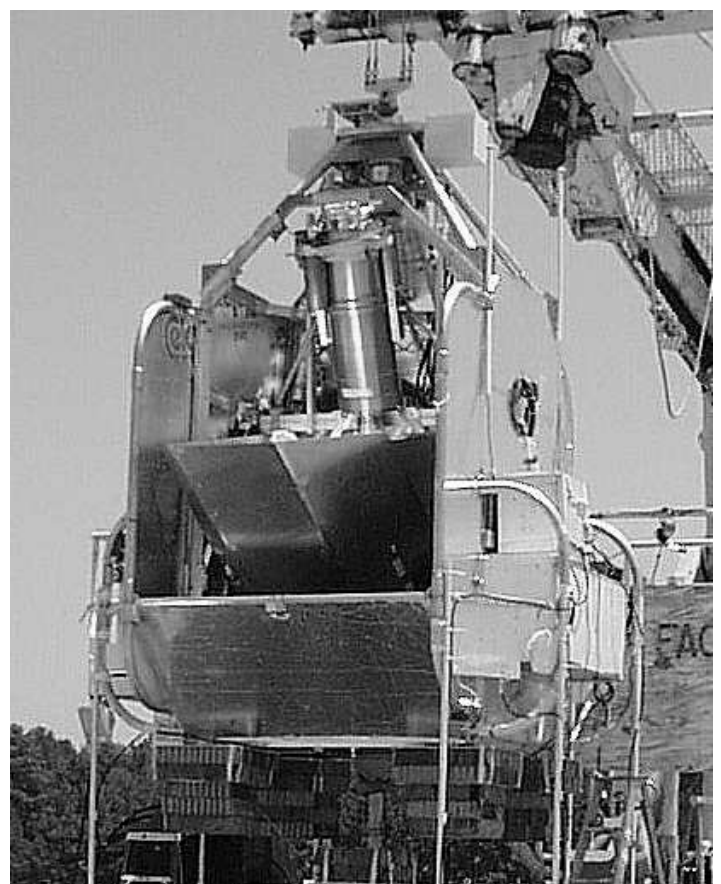

FIGURE 12. Photograph of the MAXIMA gondola shortly before launch. The receiver is visible in the center of photograph. The scoop-shaped aluminum ground shield can be seen, below the receiver. The outside of the gondola is covered with aluminum coated insulation attached to the roll-bars.

pendulation amplitude by a factor of $\approx 10$. During the MAXIMA- 1 flight the amplitude of the $0.6 \mathrm{~Hz}$ pendulation mode was an acceptable $1-10^{\prime \prime} \mathrm{rms}$.

\section{FLIGHT}

The MAXIMA-1 science flight was launched on August 2, 1998 at 00:58 UT (7:58 PM local) from the NSBF facility at Palestine Texas (see Fig. 12.). We reached a float altitude of $37.5 \mathrm{~km}$ at 4:35 UT and remained above 36.6 $\mathrm{km}$ for 3 hours and 47 minutes until the flight was terminated. The time at float was somewhat short due to the fast high-altitude winds during mid-summer. At $32 \mathrm{~km}$ we started rotating the gondola with a period of $18 \mathrm{~s}$ to measure the CMB dipole. Data from this calibration are shown in Fig 13. The dipole measurement lasted 30 minutes which included $\approx 100$ rotations. The data are well fit by a model that includes the CMB dipole, thermal emission from galactic dust, and a fraction of the signal from the $410 \mathrm{GHz}$ channel in the same row of the array. This component of the signal is attributed to emission from the atmosphere. This component decreased with time during the dipole measurement as the gondola rose from 32 to $36 \mathrm{~km}$ in altitude. It only makes a significant contribution to the first quarter of the data.

After the CMB dipole measurement, we carried out two CMB anisotropy scans which lasted a total of 3 hours. The position of one beam during this period is shown in Fig. 14. We perform three temporal modulations. First, the mirror scans $4^{\circ} \mathrm{p}-\mathrm{p}$ at $0.45 \mathrm{~Hz}$. Second, the gondola is scanned slowly in azimuth with a period of $\approx 1$ minute with the elevation of the telescope held constant. During the first and second segments the azimuth angle of the gondola scanned $8^{\circ}$ and $5.6^{\circ} \mathrm{p}$-p respectively. Finally, the telescope azimuth and elevation are changed roughly at the midpoint of the observation to rescan the same region. This second scan on the sky occurs at an angle to the first due to sky rotation producing a cross-linked pattern as shown in Fig. 14. This cross-linking of pixels allows a two dimensional map of the sky to be reconstructed from one dimensional scans in the presence of inevitable low-frequency noise in the detectors. The two-dimensional information that can be obtained from such a map gives a more sensitive measurement of the power spectrum than can be obtained from one-dimensional information. [12] The total area covered by the observation is $122 \operatorname{deg}^{2}(0.3 \%$ of the sky). This region is one of the lowest dust contrast regions of the sky based on calculations using the IRAS/DIRBE $100 \mu m$ map of Schlegel et al. [11].

During the last part of the flight we observed Jupiter to measure our beam patterns and obtain an independent calibration. We held the elevation of the telescope constant while Jupiter slowly moved through the elevation of the beams due to sky rotation. The mirror modulation provided azimuthal scans of Jupiter at many elevations. One 

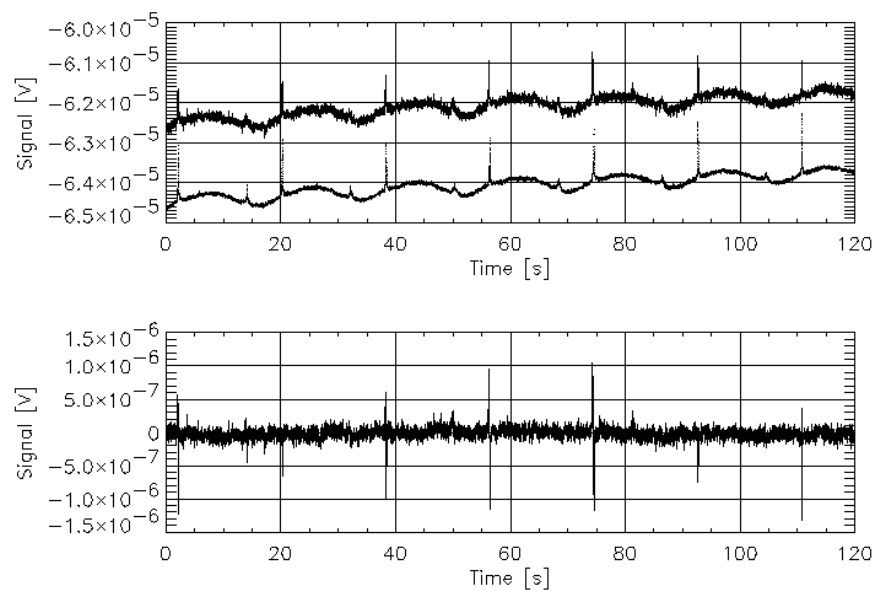

FIGURE 13. Observation of the CMB dipole during the MAXIMA-1 flight. The top panel shows raw data from one 150 $\mathrm{GHz}$ channel at the top and a model calculation at the bottom. The model is a linear combination of a dipole simulation, a galaxy simulation from an IRAS/DIRBE $100 \mu \mathrm{m}$ map [11], data from the $410 \mathrm{GHz}$ channel in the same row in the array, slope, and a constant offset. The signal correlated with the $410 \mathrm{GHz}$ channel may be due to atmospheric emission. It decreases with time during the observation as the gondola rises from 32 to $36 \mathrm{~km}$, and only makes a significant contribution to the first quarter of the data. The bottom panel shows the difference between the model and the data. The fit between model and data for this channel give a reduced $\chi^{2} \sim 1$. The galaxy crossings are not included in the fit.

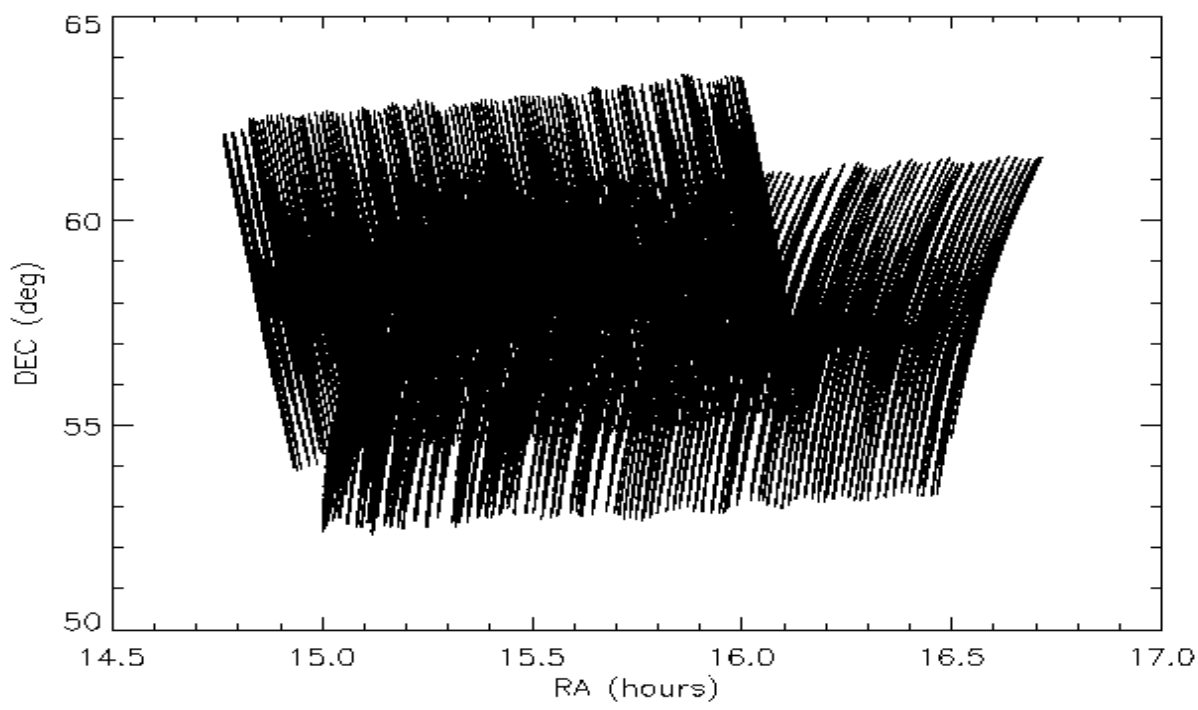

FIGURE 14. Scan pattern for MAXIMA-1 flight. Points represent the position of the beam for one channel. The total area covered by the observation is $122 \mathrm{deg}^{2}(0.3 \%$ of the sky). The duration of the observation was 3 hours. This region is one of the lowest dust contrast regions of the sky based on calculations using the IRAS/DIRBE $100 \mu \mathrm{m}$ map of Schlegel et al. [11]. Observation strategy includes three temporal modulations. The mirror scans $4^{\circ} \mathrm{p}-\mathrm{p}$ at $0.45 \mathrm{~Hz}$, the gondola is slowly sawtooth scanned in azimuth with a period of $\approx 1$ minute with the elevation of the telescope held constant, and the telescope elevation is changed roughly at the midpoint of the observation to rescan the same region.

such scan is shown in Fig. 7. We made several hundred such scans for each channel. It should be possible to form a two-dimensional map of the beam from these scans to accurately characterize the beam shapes.

The experiment was recovered after flight with little damage and is being prepared for up to two flights from 
Palestine during the summer of 1999. Data analysis from MAXIMA-1 is in progress and results will be presented in future publications.

\section{ACKNOWLEDGEMENTS}

We are grateful to R. Plambeck, R.F. Silverberg, and P. Timbie for supplying the RF sources used for the sidelobe measurements. We thank S. Church, D. Finkbeiner, E. Hivon, D. Huguenin, and S. Meyer for useful discussions. D. Horsley and J. Wu made important contributions to the attitude control system. We are very grateful to the National Scientific Balloon Facility in Palestine, Texas for their support during the MAXIMA-0 and MAXIMA-1 campaigns. This work was funded in part by NSF cooperative agreement AST-9120005 and NASA grant NAG5-4454.

\section{REFERENCES}

1. J. Bock, Ph.D. Thesis, University of California, (1997).

2. Infrared Laboratories, inc., Tucson, AZ, USA.

3. C. Hagmann and P.L. Richards, "Adiabatic Demagnetization Refrigerators for Small Laboratory Experiments and Space Astronomy," Cryogenics, Vol. 35, No. 5, pgs 303-309 (1995).

4. Eurus Monoco, inc., Minneapolis, MN, USA.

5. J.J. Bock, D. Chen, P.D. Mauskopf, and A.E. Lange, "A Novel Bolometer for Infrared and Millimeter-Wave Astrophysics," Space Science Reviews, 74, Kluwer Academic Publishers, 229 (1995)

6. S. Hanany, A.H. Jaffe, E. Scannapieco, "The Effect of the Detector Response Time on Bolometric Cosmic Microwave Background Anisotropy Experiments," Mon. Not. R. Astron. Soc. 299, pgs. 653-660 (1998).

7. T. Wilbanks, M. Devlin, A.E. Lange, S. Sato, J. W. Beeman, and E.E. Haller, "Improved Low Frequency Stability of Bolometric Detectors," IEEE Trans. Nuclear Science, vol. 37, pp. 566 (1990).

8. M. Devlin, A.E. Lange, T. Wilbanks, and S. Sato, "A DC-Coupled, High Sensitivity Bolometric Detector System for the Infrared Telescope in Space," IEEE Trans. Nuclear Science, vol. 40, pp. 162 (1993).

9. D. J. Fixsen, E. S. Cheng, D. A. Cottingham, W. C. Folz, C. A. Inman, M. S. Kowitt, S. S. Meyer, L. A. Page, J. L. Puchalla, J. E. Ruhl, and R. F. Silverberg, "A Balloon-Borne Millimeter-Wave Telescope for Cosmic Microwave Background Anisotropy Measurements," Ap.J. 47063 (1996).

10. Geneva Observatory, University of Geneva, Sauverny, Versoix, CH-1290.

11. D. J. Schlegel, D. P. Finkbeiner, and Marc Davis, "Maps of Dust IR Emission for Use in Estimation of Reddening and CMBR Foregrounds," Astrophysical Journal 500, 525 (1998) (Also astro-ph/9710327)

12. M. Tegmark, "CMB mapping experiments: a designer's guide," Phys. Rev. D, 56, pgs. 4514-4529 (1997) 